\title{
Zur Kommunikation der ersten Missionare in Mexiko
}

\author{
von Willi Henkel
}

\begin{abstract}
Als die ersten Franziskanermissionare im Jahre 1524 systematisch die Missionsarbeit in Mexiko begannen, waren ihnen Sprache, Schrift, Sitten und Gebräuche der Indios gänzlich unbekannt. Sie fanden dort hochentwickelte Kulturen der Azteken und der Mayas vor, die zahlreiche andere Stämme tributpflichtig gemacht hatten. Auch die Religionen dieser Volksstämme waren ihnen gänzlich unbekannt. Dies stellte die Missionare vor fast unüberwindliche Schwierigkeiten, als sie den Indios die christliche Botschaft verkünden wollten.
\end{abstract}

\section{Die ersten Missionsversuche}

Man kann drei Etappen in der Missionspredigt und -katechese unterscheiden: die erste darf man als stumme Predigt bezeichnen. ${ }^{2}$ In diesem Zeitraum verständigten sich die Missionare mit Gesten und Zeichen. Einen Fortschritt stellte die Kommunikation durch einfache Bilder dar. Die dritte Stufe hatten sie erreicht, als sie Predigt und Katechese in den einheimischen Sprachen vortragen konnten. Dazu waren sie nach einer relativ kurzen Zeit des Aufenthaltes im Lande fähig. Aus der Literatur jener Zeit können wir leicht feststellen, mit welchem Eifer die Missionare das Studium der Sprachen und der Kulturen der Indios betrieben. ${ }^{3}$

\section{Die Bilderschrift der Azteken}

Bei den Azteken fanden die Missionare eine Bilderschrift vor, die sie schon bald auch für die Katechese gebrauchten. Die Bilder waren auf zugeschnittene Stücke von Hirschleder oder auf Papier gemalt, die bis zu einer Länge von 10 Metern und manchmal noch länger aneinandergeleimt wurden. Nach Art eines Leporelloalbums faltete man sie zusammen; an den beiden Enden hatte man Holzdeckel angebracht. Ein Maler (tlacuilo) bemalte sie auf der einen Seite oder auch auf beiden Flächen. Bei der Schrift der Azteken handelt es sich also um Bilderalben, die man mit den »Armenbibeln« des Mittelalters vergleichen kann. Mittels dieser Figuren und Symbole konnte man auch einen Vorgang darstellen. ${ }^{4}$ Die Schriftzeichen der Azteken lassen sich in folgende fünf Klassen einteilen: Zahlen des einheimischen Schriftsystems, Kalenderangaben (Jahre, Tage), Bilder von Gegenständen, Personen und Göttern, Ideen und Symbole, phonetische Zeichen. ${ }^{5}$

\section{Die Verwendung der Bilderschrift bei den Missionaren}

Die Missionare behielten zahlreiche Schriftzeichen aus der heidnischen Zeit bei. Sie mußten freilich auch eine Anzahl von neuen Zeichen erfinden, um die christliche Botschaft auszudrücken. Sie ließen diese auf Leinwand oder auf Holz malen, als sie die Sprachen der Indios noch nicht verstanden und erläuterten sie mit Gesten. Eine weitere Art waren die oben genannten Bilderalben, die in Form von Büchern oder Codizes

Prof. Dr. Willi Henkel omi ist Direktor der Universitätsbibliothek und Professor für Missionswissenschaft an der Päpstlichen Universität Urbaniana in Rom. 
dargeboten wurden. ${ }^{6}$ Aber auch als sie die einheimischen Sprachen schon beherrschten, gebrauchten die Missionare immer noch Bildertafeln, die sie mündlich erklärten. Die Bildersprache erwies sich als sehr geeignet, um den christlichen Glauben leichter dem Gedächtnis einzuprägen.

Mit Hilfe von Bildern hörten die Missionare auch die Beichten der Neuchristen. ${ }^{7}$ Diese halfen ihnen, verhältnismäßig schnell zu einer Verständnigung zu kommen.

Die Schriftzeichen, welche die Missionare verwandten, waren nicht immer und überall gleich. So gebrauchte z.B. Jocobo de Testera O.F.M. eine Schrift, die nach ihm benannt wurde. ${ }^{8}$ Der Dominikaner Gonzalo Lucero verwandte eigene Zeichen bei seiner Missionstätigkeit unter den Mixteken. ${ }^{9}$ Der Historiker Gerónimo de Mendieta weiß über die Erfolge, die mit dieser Methode erzielt wurden, zu berichten, wenn er schreibt: "Wo man den Katechismus (Doctrina cristiana) mit Hilfe von Bildern gelehrt hat, haben die Indios dieser Pueblos ihn besser verstanden, und der Glaube wurde bei ihnen tiefer verwurzelt. $\ll^{10}$ Besondere Beachtung verdient der berühmte Bilderkatechismus des Franziskanerlaienbruders Peter von Gent. ${ }^{11}$

\section{Der Bilderkatechismus von Peter von Gent}

Von diesem wertvollen Dokument sind drei Exemplare erhalten geblieben: eines befindet sich in der Nationalbibliothek von Madrid, ein zweites im Historischen Nationalarchiv in Madrid und das dritte in der Sammlung Icazbalceta in Mexiko. ${ }^{12}$

Der Katechismus, dessen Maße 5,5 x 7,7 cm betragen, besteht aus 42 Blättern (Folios), die auf beiden Seiten beschrieben sind. ${ }^{13}$ Die Bilderzeichen werden in fünf horizontalen Reihen dargestellt. Auf der Seite 42 und 83 liest man den Namen des Verfassers, Peter von Gent. ${ }^{14}$

Inhaltlich werden folgende Themen dargestellt: ${ }^{15}$ Es beginnt mit den Gebeten (S. 421): Kreuzzeichen, Vaterunser, Ave Maria, Glaubensbekenntnis, Salve Regina, auf die die Generalbeichte folgt. Auf den Seiten 22-35 werden die Glaubensartikel behandelt und auf den Seiten 35-47 wird eine kurze Erklärung des Glaubens gegeben. Darauf folgen die Gebote Gottes (S. 47-52), die Gebote der Kirche (S. 53-55) und die Sakramente (S. 55-59). Den Abschluß des Katechismus bilden die Werke der Barmherzigkeit (S. 59-65). Nach zwei unbeschriebenen Seiten (66-67) folgt im Anhang eine Zusammenfassung der Sakramente und der Werke der Barmherzigkeit (S. 68-81) ${ }^{16}$ Fachleute schätzen, daß das Werk zwischen 1525 und 1528 entstanden ist.

Peter von Gent hatte nicht die Absicht, eine wissenschaftliche, christliche Bilderschrift zu erfinden. Er verfolgte vielmehr praktische Zwecke beim Schreiben seines Katechismus. Soweit es ihm möglich war, behielt er die Zeichen der aztektischen Bildersprache bei, die viele Indios kannten. Für christliche Bezeichnungen von Personen, Sachen und Begriffen wählte er leichtverständliche Zeichen aus, bei deren Gebrauch er jedoch nicht immer konsequent war. Zeichen, deren Bedeutung feststeht, nennt man Schlüssel. ${ }^{17}$ Die meisten von diesen können ohne weiteres verstanden werden. Einige lassen sich aus dem inhaltlichen Zusammenhang des Katechismus deuten.

Huonder bemerkt, daß die Auswahl der Zeichen manchmal »überraschend glücklich ist, oft auch recht kindlich ${ }^{18}$. So verwendet Peter von Gent für die Auferstehung Jesu Christi am dritten Tag nach dem Tod drei übereinandergestellte Sonnen, welche die 
drei Tage andeuten. Die Himmelfahrt Christi stellt er mit einer Leiter dar, auf der Christus hinaufsteigt; über dieser steht das Zeichen für Himmel. Das achte Gebot wird durch eine Gruppe von Menschen dargeboten, in der einer dem anderen das Zeichen für Sünde auf das Haupt legt. ${ }^{19}$

Die Vorzüge dieses Katechismus liegen auf der Hand; die Bilder erleichterten den Indios das gedächtnismäßige Einprägen der Glaubenswahrheiten. Die mündliche Erklärung erläuterte die dargestellten Begebenheiten.

Obwohl die Bilderschrift schon bald zu einem großen Teil von der phonetischen Aufzeichnung der einheimischen Sprachen verdrängt wurde, offenbart ihre Verwendung seitens der Missionare eine große Offenheit für die Möglichkeiten kulturell gebundener Kommunikationsmittel. Peter von Gent hat in seiner 50jährigen Missionstätigkeit die einheimischen Sprachen mit ungewöhnlichem Erfolg kennengelernt. A uf vorbildliche Weise hat er den interkulturellen Dialog gepflegt, zu dem ihn die Hochschätzung der einheimischen Kulturen vortrefflich befähigte.

Schließlich soll auf weitere Formen der Kommunikation dieses großen Missionars hingewiesen werden. Dafür kam ihm seine vielseitige Begabung zustatten. Peter von Gent beherrschte zahlreiche Musikinstrumente und lernte den Gebrauch von einheimischen dazu. Er leitete Chöre von Indios. Bei den Indios fand er eine große Wertschätzung der Musik, die ihm den Zugang zu ihrer Kultur erleichterte. Er war ein beliebter Lehrer und ein tüchter Meister der praktischen Handwerke. Er führte Prozessionen und Bruderschaften ein. Die Inschrift auf seiner Statue bei der Kathedrale von Mexiko erinnert den Besucher daran, daß Peter von Gent mit Recht als Begründer der Pädagogik in der Neuen Welt betrachtet wird. ${ }^{20}$

Einige Zeichen aus der Bilderschrift des Peter von Gent:

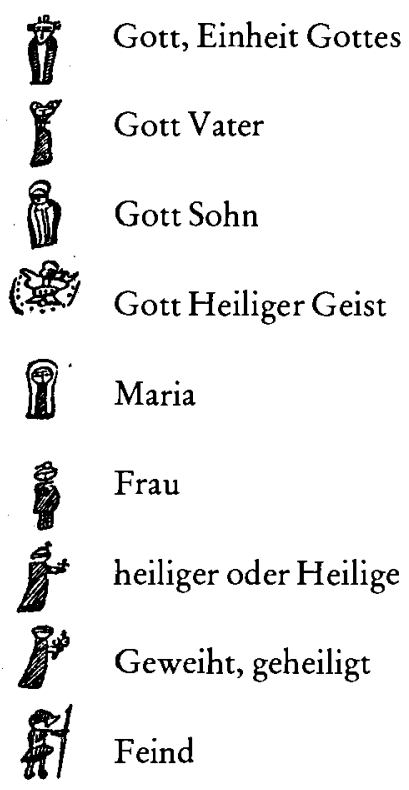

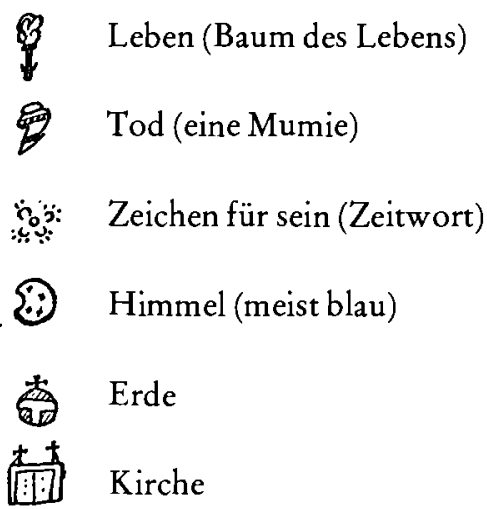

Sünde (Giftfrucht oder Skorpion?)

Strafe, Übel, Schmerz (Dolch)

Leiden, Schmerz, Passion Christi (Geissel) 
Lohn, Bezahlung, Schuld (auf alten Steuerlisten)

\section{[1.70 Name}

to Glaube (»Ich glaube «)

Der, die, das (Die Mehrzahl wird durch Wiederholung ausgedrückt)

In, bei, auf, zu (Ort und Richtung)

Und (Bindewort)
Rechts, rechte Seite

Erlösung

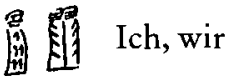

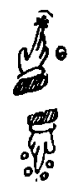

Welcher (Beziehungswort)

Heute, jetzt

Amen (am Schluß

eines Gebetes)

Aus: Die Katholischen Missionen 1917/1918, S. 170

\section{Anmerkungen:}

1 Vgl. Geronimo de Mendieta O.F.M., Historia Eclesiástica Indiana Bd. II (Mexiko 1945) S. 55-57. R. Ricard, La »conquête spirituelle« du Mexique (Paris 1933) S. 43-53.

2 J. Guillermo Durán, Monumenta Catechitica Hispanoamericana (siglos XVI-XVIII) Bd. I (Buenos Aires 1984) S. 73.

3 R. Streit, Bibliotheca Missionum Bd. II (Aachen 1924) S. 51-110.

4 W. Krickeberg, Altmexikanische Kulturen (Berlin 1956) S. 265. Neben dieser Ideenschrift gab es auch schon eine Wortschrift.

5 Durán, Monumenta Catechetica S. 96.

6 Durán, Monumenta Catechetica S. 102.

7 Durán, Monumenta Catechetica S. 103.

8 Durán, Monumenta Catechetica S. 106.

9 Durán, Monumenta Catechetica S. 110-111.

10 Durán, Monumenta Catechetica S. 112.

11 Peter von Gent (de Mura, de Moor, van der Moere, de Muer) wurde 1486 (nach anderen 1480) zu Ayghem (oder Iguen) bei Gent geboren. Wie aus seinen Briefen hervorgeht, stand er im verwandtschaftlichen Verhältnis zu Karl V. Er trat als Laienbruder in den Franziskanerorden ein. Am 27. April 1522 ging er nach Mexiko. Hier wurde er einer der bedeutendsten Missionare, der sich besondere Verdienste um die Erziehung der Jugend erwarb. Er starb 1572 in Mexiko. Vgl. Streit, Bibliotheca Missionum II, S. 62. - Eine Biographie schrieb F. Kieckens, S.J., Les anciens missionaires belges en Amérique. Fray Pedro de Gante. Recollet flamand, premier missionaire de Anahuac 1522-1572 (Bruxelles 1880).

12 Durán, Monumenta Catechetica S. 114.

13 Durán, Monumenta Catechetica S. 114.

14 Durán, Monumenta Catechetica S. 115.

15 Durán, Monumenta Catechetica S. 115.

16 Durán, Monumenta Catechetica S. 115.

17 A. Huonder, S.J., Ein merkwürdiger Missionskatechismus des 16. Jahrhunderts, in: Die Katholischen Missionen 46 (1917/18) S. 170. Huonder gibt eine Zusammenfassung der Forschungsergebnisse von Narciso Sentenach, Catecismos de la Doctrina Cristiana en Jeroglificos para ensenanza de los Indios Americanos, in: Revista de Archivos, Bibliotecas y Museos (Madrid 1900) S. 599ff. 
18 Huonder, Ein merkwürdiger Missionskatechismus S. 170.

19 Huonder, Ein merkwürdiger Missionskatechismus S. 170.

20 L. Campos, O.F.M., Fray Pedro de Gante, fundador de la pedagogía en América (14801572), in: El Clero y las Misiones 1 (Roma 1939) S. 226-235.

\section{Summary}

The communication methods of the first missionaries in Mexico developed from signlanguage to pictoral and finally local language communication of the aztecs and Mayas. The already existing pictorial language of the Aztecs was used an extended for catechesis. Missionaries, however, also had to introduce additional signs. An example of especially good use of local communication means was given by Peter van Gent who also developed a picture catechism.

\section{Résumé}

Les méthodes de communications de premiers missionaires au Mexique se développaient de la langue de signes, en passant par les figures jusqu'à l'utilisation des langues autochtones des Aztèques et des Mayas. L'écriture sous forme de figures, qui existait déjà, s'était qualifiée surtout pour le cathéchisme, mais devrait être complétée par un nombre de nouveaux de signes de figures. Peter von Gent, qui publia entr'autre aussi un chatéchisme de figures, donna surtout un exemple d'une bonne utilisation des moyens de communications existants.

\section{Resumen}

Los métodos de comunicación de los primeros misioneros en Méjico se desarrollaron del lenguaje por senas y cuadros en utilizar las lenguas nativas de los aztecas y mayas. La escritura ideográfica ya existente de los aztecas sirvió especialmente para la catequesis, pero tenia que ser completada por un numero de nuevos signos ilustrados. Peter von Gent dio un ejemplo para la utilización excelente de los medios de comunicación existentes, entre otros publicó un catecismo ilustrado. 